\title{
Correction: Single-cell profiling reveals the trajectories of natural killer cell differentiation in bone marrow and a stress signature induced by acute myeloid leukemia
}

Adeline Crinier ${ }^{1}$, Pierre-Yves Dumas ${ }^{2,3,4}$, Bertrand Escalière ${ }^{1}$, Christelle Piperoglou ${ }^{5}$, Laurine Gil ${ }^{1}$, Arnaud Villacreces ${ }^{3,4}$, Frédéric Vély ${ }^{1,5}$, Zoran Ivanovic ${ }^{4,6}$, Pierre Milpied ${ }^{1}$, Émilie Narni-Mancinelli (iD ${ }^{1 凶}$ and Éric Vivier (DD $^{1,5,7 凶}$

(c) The Author(s), under exclusive licence to CSI and USTC 2021

Cellular \& Molecular Immunology (2021) 18:2578-2580; https://doi.org/10.1038/s41423-021-00771-z

Correction to: Cellular \& Molecular Immunology https://doi.org/ 10.1038/s41423-020-00574-8, published online 25 November 2020

In the version of this article initially published, two unintended errors were made during manuscript preparation.

(1) The figure legend for Panel 3B was missing; the correct legend is as follows:

Figure 3. A Heatmap of hNK1 and hNK2 gene expression programs for each of the three healthy human bone marrow NK cell subsets. B Heatmap for CD56 ${ }^{\text {dim }}$ and CD56 $6^{\text {bright }}$ (from Hanna et al. ${ }^{10}$ ) gene expression programs for each of the three healthy human bone marrow NK cell subsets. C Heatmap for hNK_Sp1, hNK_Sp2, hNK_Sp3, and hNK_Sp4 gene expression programs for each of the three healthy human bone marrow NK cell subsets. D Flow cytometry analysis of healthy bone marrow NK cells, showing CD52, CD160, and CD56 cell surface expression in a representative healthy donor. E Frequencies of the indicated cell populations among total bone marrow NK cells from five healthy donors. F FACS profiles of the indicated cell surface marker in CD56 dim, CD56 bright $\mathrm{CD}_{160}{ }^{+} \mathrm{CD}_{2} 2^{-}$and $\mathrm{CD}_{6}{ }^{\text {bright }} \mathrm{CD} 160^{-} \mathrm{CD}^{+} 2^{+}$cell subsets in a representative healthy donor. $\mathbf{G}$ Mean fluorescence intensity of the cell surface markers shown in (F) for each of the five healthy donors. Statistical significance was assessed by Friedmann analysis with Dunn post hoc tests of paired subset measurements, and $p$ values were adjusted with the Benjamini-Hochberg method. Error bars indicate the mean $( \pm \mathrm{SD}) .{ }^{*} p$ value $<0.05,{ }^{* *} p$ value $<0.01$, ${ }^{* * *} p$ value $<0.001,{ }^{* * *} p$ value $<0.0001$.

(2) Supplementary Table S2 and the corresponding legend were missing: Supplementary Table S2. A Selected characteristics of the healthy donors, including sex, age, and serological status for CMV analyzed by scRNA-seq. B Selected characteristics of AML patients, including sex, age, FAB classification, AML status, percentage of blasts in the bone marrow and serological status for CMV and EBV analyzed by scRNA-seq. C Selected characteristics of the healthy donors, including sex, age and serological status for CMV analyzed by flow cytometry. D Selected characteristics of the AML patients including sex, age, FAB classification, AML status, percentage of blasts in the bone marrow and serological status of CMV and EBV analyzed by flow cytometry. NOS: not otherwise specified; trAML: therapy-related $A M L ; C T$ : chemotherapy; $R T$ : radiation therapy; sAML: secondary AML; PV: polycythemia vera; CML: chronic myeloid leukemia; PMF: primary myelofibrosis; NA: not available.

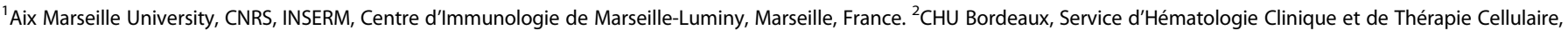

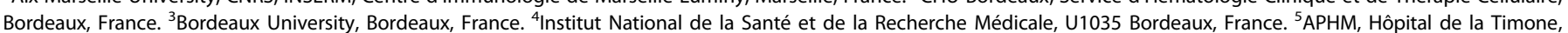

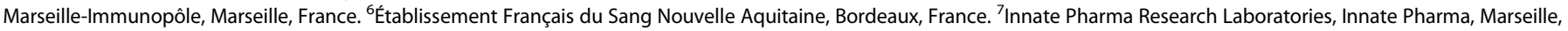
France. ${ }^{凶}$ email: narni@ciml.univ-mrs.fr; vivier@ciml.univ-mrs.fr

Published online: 29 September 2021 
A.

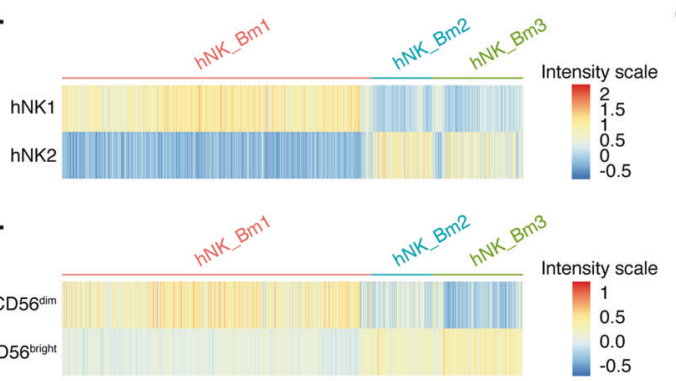

D.

On human bone marrow lymphocytes $\mathrm{CD} 45^{+}$alive
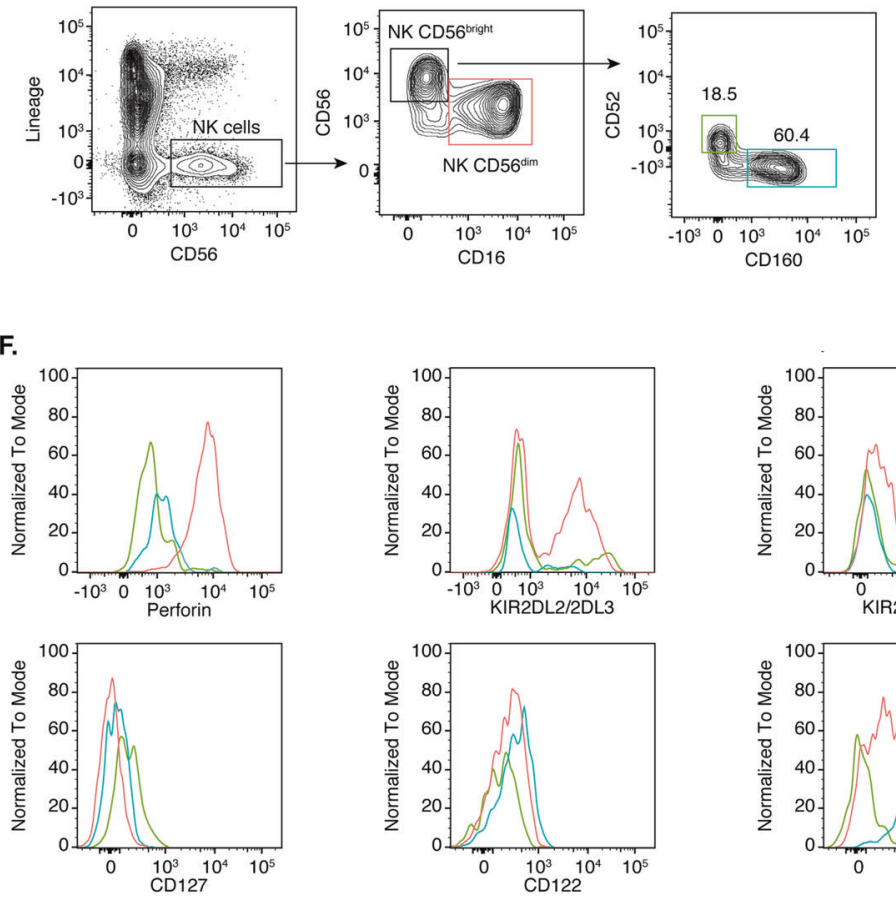

G.
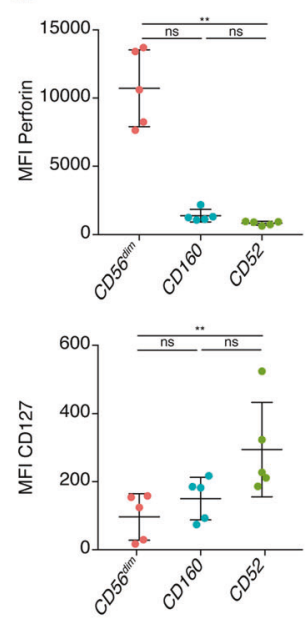
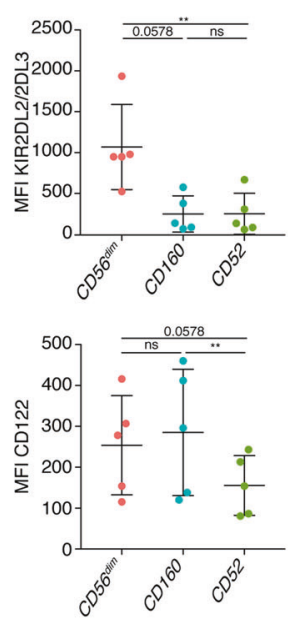

C.

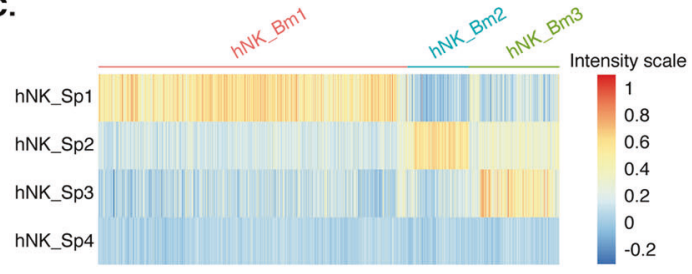

E.

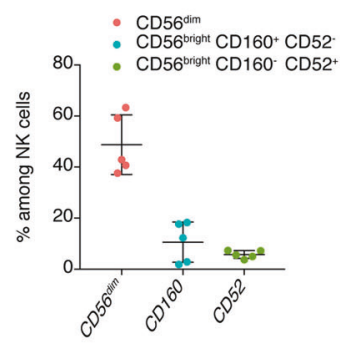

Fig. 3

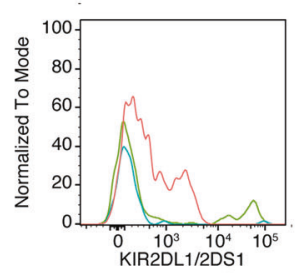

- CD56 dim

CD56 ${ }^{\text {brigh }} \mathrm{CD} 160^{+} \mathrm{CD} 52$ - CD56 bright CD160. CD52+
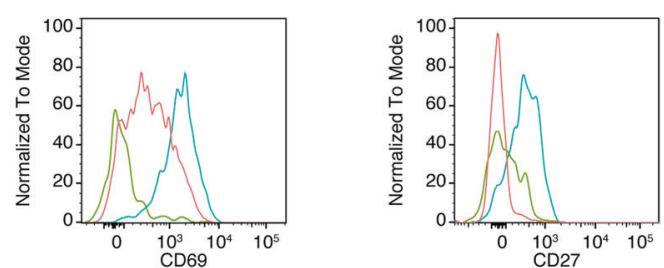

- $\mathrm{CD}^{\mathrm{C}} 6^{\text {dim }} 6^{\text {tright }} \mathrm{CD} 160^{+} \mathrm{CD} 52$ - CD56 ${ }^{\text {bright }}$ CD160 $0^{\circ}$ CD52
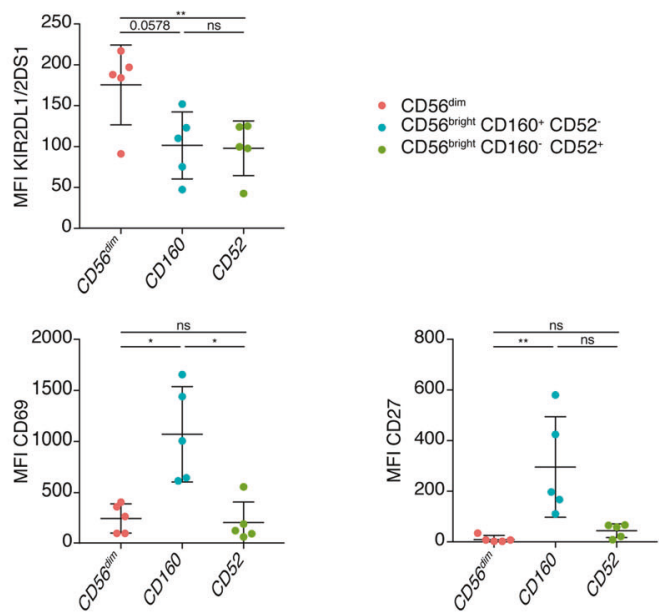
A. Crinier et al.

2580

ADDITIONAL INFORMATION

Supplementary information The online version contains supplementary material available at https://doi.org/10.1038/s41423-021-00771-z.

Correspondence and requests for materials should be addressed to Émilie NarniMancinelli or Éric Vivier.

Reprints and permission information is available at http://www.nature.com/ reprints 